May 7, 2009 11:10 WSPC/INSTRUCTION FILE main

Discrete Mathematics, Algorithms and Applications

(C) World Scientific Publishing Company

\title{
Analyzing Nonblocking Multilog Networks With the König-Egeváry Theorem
}

\author{
Hung Q. Ngo* \\ Computer Science and Engineering, State University of New York at Buffalo, 201 Bell Hall \\ Amherst, New York, The United States of America, \\ hungngo@cse.buffalo.edu \\ Thanh-Nhan Nguyen \\ Computer Science and Engineering, State University of New York at Buffalo, 201 Bell Hall \\ Amherst, New York, The United States of America, \\ nguyen9@cse.buffalo.edu \\ Duc T. Ha \\ Computer Science and Engineering, State University of New York at Buffalo, 201 Bell Hall \\ Amherst, New York, The United States of America, \\ ducha@cse.buffalo.edu
}

\begin{abstract}
When analyzing a nonblocking switching network, the typical problem is to find a route for a new request through the network without disturbing existing routes. By solving this problem, we can derive how many hardware components of a certain type (Banyan planes in a multi-log network, for instance) are needed for the network to be nonblocking. This scenario appears in virtually all combinations of switching environments: strictly, widesense or rearrangeably nonblocking, unicast or multicast switching, and circuit, multirate, or photonic switching.

In this paper, we show that the König-Egevarý theorem is a very good tool which helps solve the above prototypical problem. The idea is to somehow "represent" the potential blocking connections as edges of a bipartite graph. The maximum number of blocking connections roughly corresponds to the size of a maximum matching in that bipartite graph. The size of any vertex cover, by the König-Egevarý theorem, is an upper bound on the maximum number of blocking connections. Thus, by specifying a small vertex cover, we can derive the sufficient number of hardware components for the network to be nonblocking. We illustrate the technique by analyzing crosstalk-free and non-crosstalk-free widesense nonblocking multicast multi-log networks.

Particularly, for the first time in the literature we derive conditions for the $d$-ary multi-log network to be crosstalk-free multicast widesense nonblocking under the window algorithm for any given window size. Several by-products follow from our approach and results. Firstly, our results allow for computing the best window size minimizing the fabric cost, showing that the multi-log network is a good candidate for crosstalk-free multicast switching architectures. Secondly, the analytical approach also gives a much simpler proof of the known case when the network is not required to be crosstalk-free. Thirdly, we show that several known results for the multi-log multicast networks under the so-called fanout constraints are simple corollaries of our results.
\end{abstract}

${ }^{*}$ Hung Q. Ngo is supported in part by NSF CAREER Award CCF 0347565. 


\section{Introduction}

\subsection{The crosstalk-free widesense nonblocking photonic switching problem}

Researchers in recent years have spent a lot of effort on analyzing and designing photonic switching architectures at all levels of granularities: from space, wavelength, down to the optical-burst/packet level [8,15, 17, 18,20,26,28,29]. Whichever the switching-granularity is, space-switching architectures often provide the design guidance. (For example, at the wavelength-level, wavelength converters are placed at appropriate places in a space-domain switch.) Moreover, multicasting is certainly a fundamental communication primitive. Consequently, designing cost-effective multicast-capable photonic switching networks (or optical cross-connects) in the space domain is a fundamental problem in optical switching.

The switching elements (SE) of a photonic switching network can naturally be constructed with perhaps the most widely studied and commercially available optical component: the directional couplers [14,22,27] (for the $2 \times 2$-SE case). However, directional couplers and many other optical SEs suffer from optical crosstalk between interfering channels, which is one of the major obstacles in designing cost-effective switches [1,3,24]. Although crosstalk is a complicated phenomenon to model, it has been shown that reducing the number of SEs shared between active routes in a switch will reduce crosstalk. In particular, if no two active routes share any common SE, then the design is said to be crosstalk-free [13,25].

There are three levels of nonblockingness of a switch: rearrangeably nonblocking (RNB), widesense nonblocking (WSNB), and strictly nonblocking (SNB). The reader is referred to [6] for their precise definitions. SNB switches in principle require minimal effort in routing a new request at the price of higher switch cost (in terms of the number of SEs). RNB switches have the lowest cost but require rearranging existing connections, complicating route establishment algorithms, and disrupting existing traffic flows. WSNB switches strike a balance between SNB and RNB switches, both in terms of cost-effectiveness and routing-establishment efficiency.

One of our main results is on the problem of designing cost-effective crosstalk-free WSNB multicast photonic switching networks.

\subsection{The multi-log architecture and its analysis}

The multi-log architecture is a good choice for designing photonic switching networks, because they have small depth $(O(\log N))$, absolute signal loss uniformity, and good fault tolerance $[5,8,10,11,13,21,25]$. Henceforth, let $\log _{d}(N, 0, m)$ denote a $d$-ary multi-log network with $m$ vertically stacked inverse Banyan planes (denoted by BY $\mathrm{B}^{-1}(n)$ ), where $N=d^{n}$ is the number of inputs/outputs of the network. See Figure 1 for an illustration. Figure 2 gives an illustration of an inverse Banyan network, and Figure 3 depicts a particular multi-log network - the $\log _{3}(27,0,2)$ network.

There have been many prior works analyzing the multi-log networks. For example, various necessary and/or sufficient conditions have been derived for the $\log _{d}(N, 0, m)$ networks to be $f$-cast SNB [26], multicast WSNB [2, 4], $f$-cast RNB [7, 19], unicast SNB under various crosstalk constraints $[25,26]$. These are only a small sample of results on 


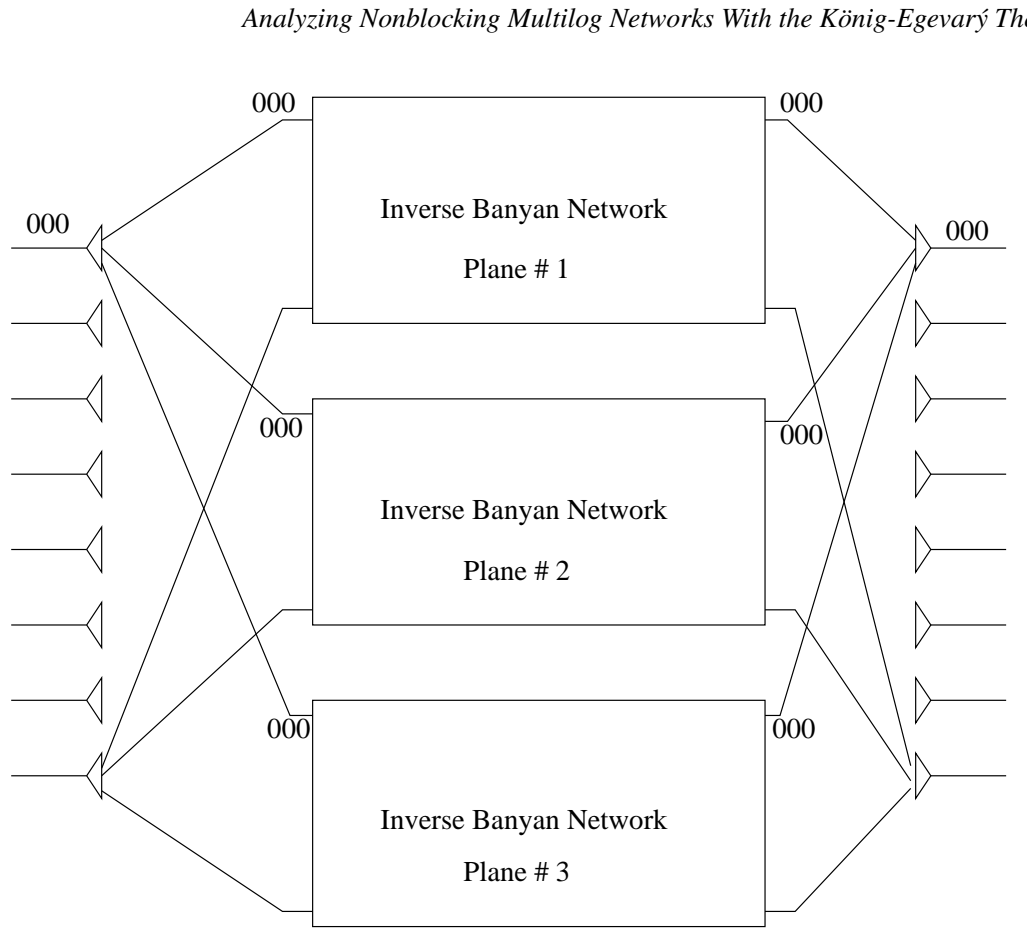

Fig. 1. The basic structure of the multi-log network with 3 inverse Banyan planes

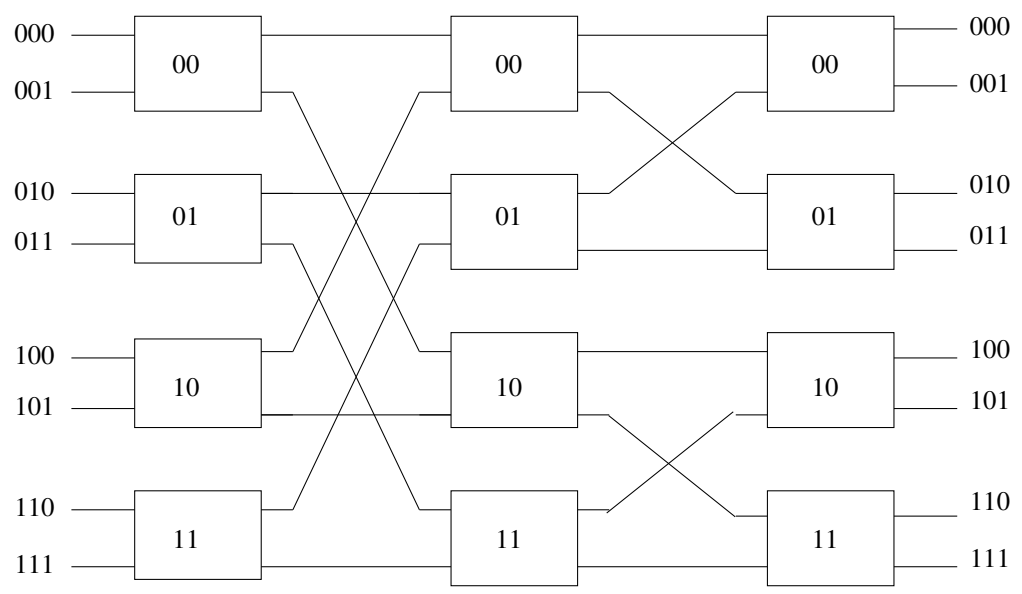

Fig. 2. The inverse Banyan network $\mathrm{BY}^{-1}(3)$

multi-log networks. The reader is referred to the above papers and references thereof.

Most existing analytical approaches of the multi-log networks are similar: they are based on tedious counting arguments involving the blocking connections. The drawback of this line of argument is that the proofs are often long and their correctness are not easy to 


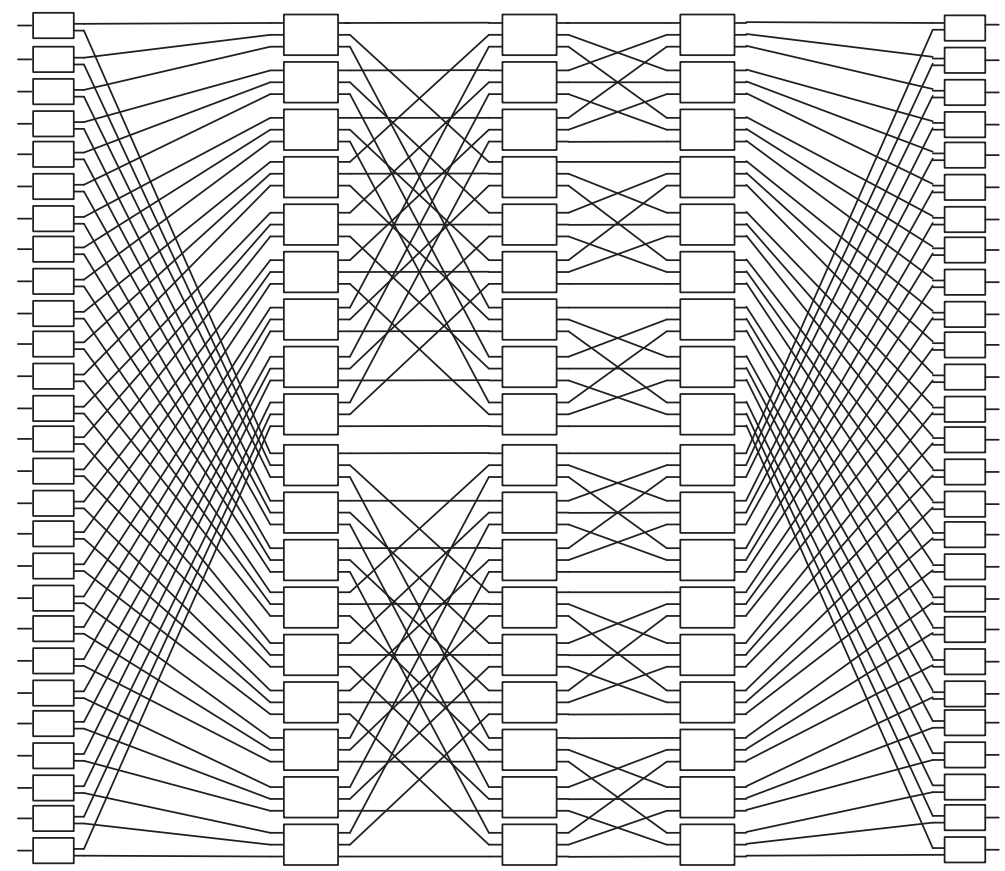

Fig. 3. The $\log _{3}(27,0,2)$ network

verify. We will show that the classic König-Egevarý theorem is a very good analytical tool for this problem.

The main idea can briefly be outlined as follows. We somehow "represent" the potential blocking connections as edges of a bipartite graph. The maximum number of blocking connections roughly corresponds to the size of a maximum matching in that bipartite graph. The size of any vertex cover, by the König-Egevarý theorem, is an upper bound on the maximum number of blocking connections. By specifying any small vertex cover and computing its size, we can derive the sufficient number of hardware components for the network to be nonblocking. We illustrate the technique by analyzing crosstalk-free and non-crosstalk-free widesense nonblocking multicast multi-log networks.

\subsection{Main results and paper outline}

We derive conditions for the $d$-ary multi-log network to be crosstalk-free multicast widesense nonblocking under the window algorithm [9,23] for any given window size. Several by-products follow from our approach and results. Firstly, our results allow for computing the best window size minimizing the fabric cost, showing that the multi-log network is a good candidate for crosstalk-free multicast switching architectures. Secondly, the analytical approach also gives a much simpler proof of the known case when the network is not required to be crosstalk-free. Thirdly, we show that several known results (in [26]) for 
May 7, $2009 \quad$ 11:10 WSPC/INSTRUCTION FILE main

the multi-log multicast networks under the so-called fanout constraints are simple corollaries of our results.

The rest of the paper is organized as follows. Section 2 presents basic notations, the window algorithm, and a simple algebraic view of $\log _{d}(N, 0, m)$ networks, which are used throughout the paper. Section 3 derives conditions for $\log _{d}(N, 0, m)$ to be crosstalk-free multicast WSNB, along with several consequences including computing the optimal window size. Section 4 concludes the paper with a few remarks regarding our proof technique and its application in deriving conditions where crosstalk-free is not required.

\section{Preliminaries}

Throughout this paper, for any positive integers $k, d$, let $[k]$ denote the set $\{1, \ldots, k\}, \mathbb{Z}_{d}$ denote the set $\{0, \ldots, d-1\}$ which can be thought of as $d$-ary "symbols", $\mathbb{Z}_{d}^{k}$ denote the set of all $d$-ary strings of length $k, b^{l}$ denote the string with symbol $b \in \mathbb{Z}_{d}$ repeated $l$ times (e.g., $\left.3^{4}=3333\right),|\mathbf{s}|$ denote the length of any $d$-ary string $\mathbf{s}\left(\right.$ e.g., $|31|=2$ ), $\mathbf{s}_{i . . j}$ denote the substring $s_{i} \cdots s_{j}$ of a string $\mathbf{s}=s_{1} \ldots s_{l} \in \mathbb{Z}_{d}^{l}$ (if $i>j$ then $\mathbf{s}_{i . . j}$ is the empty string).

Let $N=d^{n}$. We consider the $\log _{d}(N, 0, m)$ network, which denotes the stacking of $m$ copies of the $d$-ary inverse Banyan network $\mathrm{BY}^{-1}(n)$ with $N$ inputs and $N$ outputs. Label the inputs and outputs of $\mathrm{BY}^{-1}(n)$ with $d$-ary strings of length $n$. Specifically, each input $\mathbf{x} \in \mathbb{Z}_{d}^{n}$ and output $\mathbf{y} \in \mathbb{Z}_{d}^{n}$ have the form $\mathbf{x}=x_{1} \cdots x_{n}, \mathbf{y}=y_{1} \cdots y_{n}$, where $x_{i}, y_{i} \in \mathbb{Z}_{d}$, $\forall i \in[n]$.

Also, label the $d \times d$ SEs in each of the $n$ stages of $\mathrm{BY}^{-1}(n)$ with $d$-ary strings of length $n-1$. An input $\mathbf{x}$ (respectively, output $\mathbf{y}$ ) is connected to the SE labeled $\mathbf{x}_{1 . . n-1}$ in the first stage (respectively, $\mathbf{y}_{1 . . n-1}$ in the last stage).

For the sake of clarity, let us first consider a small example. Consider the unicast request $(\mathbf{x}, \mathbf{y})=(011,101)$ when $d=2, n=3$. (See Figure 2 for an illustration.) The input $\mathbf{x}=011$ is connected to the SE labeled 01 in the first stage, which is connected to two SEs labeled 01 and 11 in the second stage, and so on. The unique path from $\mathbf{x}$ to $\mathbf{y}$ in the $\mathrm{BY}^{-1}(n)$-plane can be explicitly written out:

\begin{tabular}{l|l} 
input $\mathbf{x}$ & 011 \\
\hline stage-1 SE & 01 \\
stage-2 SE & $\mathbf{1 1}$ \\
stage-3 SE & $\mathbf{1 0}$ \\
\hline output $\mathbf{y}$ & 101
\end{tabular}

We can see clearly the pattern: the prefixes of $\mathbf{y}_{1 . . n-1}$ are "taking over" the prefixes of $\mathbf{x}_{1 . . n-1}$ on the path from $\mathbf{x}$ to $\mathbf{y}$. In general, the unique path $R(\mathbf{x}, \mathbf{y})$ in a $\mathrm{BY}^{-1}(n)$-plane from an arbitrary input $\mathbf{x}$ to an arbitrary output $\mathbf{y}$ is exactly the following: 


\begin{tabular}{c|l} 
input $\mathbf{x}$ & $x_{1} x_{2} \ldots x_{n-1} x_{n}$ \\
\hline stage-1 SE & $x_{1} x_{2} \ldots x_{n-1}$ \\
stage-2 SE & $y_{1} x_{2} \ldots x_{n-1}$ \\
stage-3 SE & $y_{1} y_{2} \ldots x_{n-1}$ \\
$\vdots$ & $\vdots$ \\
stage- $n$ SE & $y_{1} y_{2} \ldots y_{n-1}$ \\
\hline output $\mathbf{y}$ & $y_{1} y_{2} \ldots y_{n-1} y_{n}$
\end{tabular}

Now, consider two unicast requests $(\mathbf{a}, \mathbf{b})$ and $(\mathbf{x}, \mathbf{y})$. From the observation above, routes $R(\mathbf{a}, \mathbf{b})$ and $R(\mathbf{x}, \mathbf{y})$ share a common SE if and only if there is some $j \in[n]$ such that $b_{1 . . j-1}=y_{1 . . j-1}$ and $a_{j . . n-1}=x_{j . n-1}$. In this case, the two routes intersect at a stage- $j$ SE. (Note: two requests' routes may intersect at more than one SE.)

For any two $d$-ary strings $\mathbf{u}, \mathbf{v} \in \mathbb{Z}_{d}^{l}$, let $\operatorname{PRE}(\mathbf{u}, \mathbf{v})$ denote the longest common prefix, and $\operatorname{SUF}(\mathbf{u}, \mathbf{v})$ denote the longest common suffix of $\mathbf{u}$ and $\mathbf{v}$, respectively. For example, if $\mathbf{u}=0100110$ and $\mathbf{v}=0101010$, then $\operatorname{PRE}(\mathbf{u}, \mathbf{v})=010$ and $\operatorname{SuF}(\mathbf{u}, \mathbf{v})=10$. The following proposition immediately follows.

Proposition 2.1. Let $(\mathbf{a}, \mathbf{b})$ and $(\mathbf{x}, \mathbf{y})$ be two unicast requests. Their routes $R(\mathbf{a}, \mathbf{b})$ and $R(\mathbf{x}, \mathbf{y})$ in the same $B Y^{-1}(n)$-plane share at least a common $S E$ if and only if

$$
\left|\operatorname{SUF}\left(\mathbf{a}_{1 . . n-1}, \mathbf{x}_{1 . . n-1}\right)\right|+\left|\operatorname{PRE}\left(\mathbf{b}_{1 . . n-1}, \mathbf{y}_{1 . . n-1}\right)\right| \geq n-1 .
$$

Moreover, the routes $R(\mathbf{a}, \mathbf{b})$ and $R(\mathbf{x}, \mathbf{y})$ intersect at exactly one $S E$ if and only if

$$
\left|\operatorname{SUF}\left(\mathbf{a}_{1 . . n-1}, \mathbf{x}_{1 . . n-1}\right)\right|+\left|\operatorname{PRE}\left(\mathbf{b}_{1 . . n-1}, \mathbf{y}_{1 . . n-1}\right)\right|=n-1,
$$

in which case the common $S E$ is an $S E$ at stage $\left|\operatorname{PRE}\left(\mathbf{b}_{1 . . n-1}, \mathbf{y}_{1 . . n-1}\right)\right|+1$ of the $B Y^{-1}(n)$ plane.

The Window Algorithm: the so-called window algorithm for routing multicast requests in a multi-log network was proposed in [23] and extended in [9]. In its present form, the window algorithm with window size $d^{t}$ can be described as follows. Given any integer $t, 0 \leq t \leq n$, divide the outputs into windows of size $d^{t}$ each. Each window consists of outputs sharing a prefix of length $n-t$, for a total of $d^{n-t}$ windows. Given a new multicast request $(\mathbf{a}, B)$, where $\mathbf{a}$ is an input and $B$ is a subset of outputs, let $B_{w}$ be the set of outputs in $B$ lying in the $w$ th window. Route the sub-request $\left(\mathbf{a}, B_{i}\right)$ separately on the same $\mathrm{BY}^{-1}(n)$-plane.

Remark 2.2. Note that the window algorithm does not really specify exactly which $\mathrm{BY}^{-1}(n)$-plane(s) to route the sub-request(s) when there are more than one choice. This was the source of the confusion regarding whether the network is multicast SNB (wrongly claimed in the original paper [23]) or it is multicast WSNB (correction made in [9]). Thus, different strategies for picking available $\mathrm{BY}^{-1}(n)$-planes will be different algorithms. This is the reason we only derive sufficient conditions for the window algorithm in this paper; because, in order to derive necessary conditions one needs a particular strategy for picking available $\mathrm{BY}^{-1}(n)$-planes. 


\section{Main Results}

The following theorem is the main result of the paper. Its consequences and the proof technique's implications are explored later in this section and in Section 4.

Theorem 3.1. The d-ary multi-log network $\log _{d}(N, 0, m)$ is crosstalk-free widesense nonblocking under the window algorithm with window size $d^{t}$ if

$$
m \geq\left\{\begin{array}{l}
d^{n-2 t}+t d^{n-t}(d-1), \text { when } t<n / 2, \\
d^{n-t}[(d-1)(n-t)-1]+d^{t}+1, \text { when } t=n / 2, \\
d^{n-t}[(d-1)(n-t)-1]+d^{t}-d^{2 t-n-2}(d-1)+1 \\
\text { when } t>n / 2
\end{array}\right.
$$

Proof. Let $(\mathbf{a}, B)$ be an arbitrary multicast request to be routed using the window algorithm with window size $d^{t}$. Since each part of a multicast request belonging to the same window can be routed independently from each other, we can assume that $B=$ $\left\{\mathbf{b}^{(1)}, \ldots, \mathbf{b}^{(k)}\right\}$ where all the $\mathbf{b}^{(p)}(p \in[k])$ belong to the same window. Specifically, the $\mathbf{b}^{(p)}$ share a common prefix of length $n-t$.

For each $i \in\{0, \ldots, n-1\}$, let $A_{i}$ be the set of inputs $\mathbf{x}$ other than $\mathbf{a}$, where $\mathbf{x}_{1 . . n-1}$ shares a suffix of length exactly $i$ with $\mathbf{a}_{1 . . n-1}$. Formally, define

$$
A_{i}:=\left\{\mathbf{x} \in \mathbb{Z}_{d}^{n}-\{\mathbf{a}\} \mid \operatorname{SUF}\left(\mathbf{x}_{1 . . n-1}, \mathbf{a}_{1 . . n-1}\right)=i\right\} .
$$

For each $j \in\{0, \ldots, n-1\}$, let $B_{j}$ be the set of outputs other than those in B which share a prefix of length exactly $j$ with some member of $B$, namely

$$
B_{j}:=\left\{\mathbf{y} \in \mathbb{Z}_{d}^{n}-B \mid \exists p \in[k], \operatorname{PRE}\left(\mathbf{y}_{1 . . n-1}, \mathbf{b}_{1 . . n-1}^{(p)}\right)=j\right\} .
$$

Note that $\left|A_{i}\right|=d^{n-i}-d^{n-1-i}$, for all $0 \leq i \leq n-1$, and $\left|B_{j}\right|=d^{n-j}-d^{n-1-j}$ for all $0 \leq j \leq n-t-1$. Moreover, for each $j \leq n-t-1, B_{j}$ is the disjoint union of precisely $d^{n-j-t}-d^{n-1-j-t}$ windows of size $d^{t}$ each. When $j \geq n-t, B_{j}$ belongs to the same window which $B$ belongs.

Suppose the network $\log _{d}(N, 0, m)$ already had some routes established. Consider a $\mathrm{BY}^{-1}(n)$ plane which blocks the new request $(\mathbf{a}, B)$. There must be one route $R(\mathbf{x}, \mathbf{y})$ on this plane for which $R(\mathbf{x}, \mathbf{y})$ and $R\left(\mathbf{a}, \mathbf{b}^{(p)}\right)$ share an SE, for some $p \in[k]$. Note that $R(\mathbf{x}, \mathbf{y})$ could be part of a multicast request from input $\mathbf{x}$, but we only need the branch $(\mathbf{x}, \mathbf{y})$ of this multicast request to block $(\mathbf{a}, B)$. Assemble one blocking branch $(\mathbf{x}, \mathbf{y})$ per blocking plane into a set $S$. Then, the number of blocking planes is $|S|$. This way, if $(\mathbf{x}, \mathbf{y})$ and $\left(\mathbf{x}, \mathbf{y}^{\prime}\right)$ are both in $S$ then $\mathbf{y}$ and $\mathbf{y}^{\prime}$ must belong to different windows. We will refer to this property as the unique window property of the $(\mathbf{x}, \mathbf{y}) \in S$.

The strategy is to find and upperbound $U$ for $|S|$, where $U$ is independent of $(\mathbf{a}, B)$; then, $U+1$ will be a sufficient number of planes for the network to be nonblocking. 
By proposition 2.1, each $(\mathbf{x}, \mathbf{y}) \in S$ must belong to some $A_{i} \times B_{j}$ where $i+j \geq n-1$. Partition $S$ into two parts:

$$
\begin{aligned}
& S_{1}=\left\{(\mathbf{x}, \mathbf{y}) \in S \mid \mathbf{y} \in B_{j}, j \leq n-t-1\right\} \\
& S_{2}=\left\{(\mathbf{x}, \mathbf{y}) \in S \mid \mathbf{y} \in B_{j}, j \geq n-t\right\}
\end{aligned}
$$

To upperbound $|S|=\left|S_{1}\right|+\left|S_{2}\right|$, we bound $\left|S_{1}\right|$ and $\left|S_{2}\right|$ separately.

We first derive an upperbound for $\left|S_{1}\right|$. As noted earlier, for each $j \leq n-t-1$ there are precisely $d^{n-j-t}-d^{n-1-j-t}$ windows in $B_{j}$, each of which contains $d^{t}$ outputs. Each output $\mathbf{y}$ in a window of $B_{j}$ can only be part of at most one $(\mathbf{x}, \mathbf{y}) \in S_{1}$, because each output can only be requested once. This fact and the unique window property give the following bound:

$$
\begin{aligned}
\left|\left\{(\mathbf{x}, \mathbf{y}) \in S_{1}: \mathbf{y} \in B_{j}\right\}\right| & \leq\left(d^{n-j-t}-d^{n-1-j-t}\right) \min \left\{d^{t}, \sum_{i \geq n-1-j}\left|A_{i}\right|\right\} \\
& =\left(d^{n-j-t}-d^{n-1-j-t}\right) \min \left\{d^{t}, d^{j+1}-1\right\}
\end{aligned}
$$

Summing the above over $j=0, \ldots, n-t-1$ we obtain

$$
\begin{aligned}
\left|S_{1}\right| & =\sum_{j=0}^{n-t-1}\left|\left\{(\mathbf{x}, \mathbf{y}) \in S_{1}: \mathbf{y} \in B_{j}\right\}\right| \\
& \leq \sum_{j=0}^{n-t-1}\left(d^{n-j-t}-d^{n-1-j-t}\right) \min \left\{d^{t}, d^{j+1}-1\right\} \\
& = \begin{cases}d^{n-2 t}-d^{t}+t d^{n-t}(d-1) & t<n / 2 \\
d^{n-t}(d-1)(n-t)-d^{n-t}+1 & t \geq n / 2\end{cases}
\end{aligned}
$$

Next, we derive an upperbound for $\left|S_{2}\right|$. By definition, for each $(\mathbf{x}, \mathbf{y}) \in S_{2}$ we have $\mathbf{y} \in B_{j}$ where $j \geq n-t$. As noted before, $B_{j}$ belongs to the same window as that of $B$ for all such $j$. Construct a bipartite graph $G=(L \cup R, E)$ where $L=\bigcup_{i=0}^{n-1} X_{i}$ is the set of inputs, $R=\bigcup_{j=n-t}^{n-1} B_{j}$, and $E$ is the union of all $A_{i} \times B_{j}$ for which $j \geq n-t$ and $i+j \geq n-1$. Then, $S_{2}$ forms a matching of $G$. The classic König-Egevarý theorem [12] says that the size of a maximum matching in a bipartite graph $G$ is equal to the $\operatorname{size} \nu(G)$ of a minimum vertex cover of $G$. Thus,

$$
\left|S_{2}\right| \leq \nu(G)
$$

So, to upperbound $\left|S_{2}\right|$ we will find an upperbound for the minimum vertex cover of $G$. Note that $G$ and thus $\nu(G)$ depends on $(\mathbf{a}, B)$. We will need to find a bound independent of $(\mathbf{a}, B)$, which will be the worst-case bound.

Since the $B_{j}(j \geq n-t)$ belong to the same window, we have $|R|=d^{t}-k$. We can assume that $k<d^{t}$, otherwise the graph $G$ is empty and we reach the trivial case $\left|S_{2}\right|=0$. Let $r=\left\lfloor\log _{d} k\right\rfloor$, then $d^{r} \leq k<d^{r+1}$ and $0 \leq r \leq t-1$. Consider the following vertex 
sets of $G$, indexed by integers $q$ from $n-t$ to $n$.

$$
\begin{aligned}
C_{n-t} & =\bigcup_{j=n-t}^{n} B_{j} \\
C_{q} & =\left(\bigcup_{i=n-q}^{n-1} A_{i}\right) \cup\left(\bigcup_{j=q}^{n-1} B_{j}\right), \text { for } n-t<q \leq n
\end{aligned}
$$

Firstly, we claim that $C_{q}$ is a vertex cover of $G$ for any $q \in\{n-t, \ldots, n\}$. When $q=$ $n-t, C_{q}$ is a vertex cover since $C_{q}=R$ and every edge is incident to some vertex in $R$. When $n-t<q \leq n-1$, if there is an edge $(\mathbf{x}, \mathbf{y})$ of $G$ which is not covered by $C_{q}$ then $(\mathbf{x}, \mathbf{y}) \in A_{i} \times B_{j}$ where $i \leq n-q-1$ and $j \leq q-1$. However, in that case $i+j \leq(n-q-1)+(q-1)=n-2$, contradicting the definition of $G$. Lastly, when $q=n$, we have $C_{q}=\bigcup_{i=0}^{n-1} A_{i}=L$ and thus $C_{q}$ is certainly a vertex cover.

We already know $\left|C_{n-t}\right|=d^{t}-k$. For $n-t<q \leq n$, note that $\left|\bigcup_{j=q}^{n-1} B_{j}\right|$ is the number of outputs other than those in $B$ sharing a prefix of length at least $q$ with one of the $\mathbf{b}^{(p)}, p \in[k]$. For each $p \in[k]$, the number of outputs sharing a prefix of length at least $q$ with $\mathbf{b}^{(p)}$ (other than $\mathbf{b}^{(p)}$ itself) is at most $d^{n-q}-1$. Hence, by the union bound

$$
\left|\bigcup_{j=q}^{n-1} B_{j}\right| \leq k\left(d^{n-q}-1\right) .
$$

Thus, for $n-t<q \leq n$, we have

$$
\begin{aligned}
\left|C_{q}\right| & =\left|\bigcup_{i=n-q}^{n-1} A_{i}\right|+\left|\bigcup_{j=q}^{n-1} B_{j}\right|=d^{q}-1+\left|\bigcup_{j=q}^{n-1} B_{j}\right| \\
& \leq d^{q}-1+k\left(d^{n-q}-1\right) .
\end{aligned}
$$

To this end, consider three cases as follows. The main idea is to choose the vertex cover $C_{q_{0}}$ with minimum size depending on the relationship between $t$ and $n$.

Case 1: $2 t \leq n$. In this case, set $q_{0}=n-t$. Then,

$$
\nu(G) \leq\left|C_{n-t}\right|=d^{t}-k \leq d^{t}-1 .
$$

Case 2: $2 t \geq n+1$ and $k \geq d^{2 t-n-1}-d^{2 t-n-2}+1$. In this case, set $q_{0}=n-t$. Then,

$$
\nu(G) \leq\left|C_{n-t}\right|=d^{t}-k \leq d^{t}-d^{2 t-n-1}+d^{2 t-n-2}-1 .
$$

Case $32 t \geq n+1$ and $k \leq d^{2 t-n-1}-d^{2 t-n-2}$. This means $r \leq 2 t-n-2$.

Case 3a If $r=2 t-n-2$, then setting $q_{0}=t-1$ we get

$$
\begin{aligned}
\nu(G) & \leq\left|C_{t-1}\right| \leq d^{t-1}-1+k\left(d^{n-t+1}-1\right) \\
& \leq d^{t-1}-1+\left(d^{2 t-n-1}-d^{2 t-n-2}\right)\left(d^{n-t+1}-1\right) \\
& =d^{t}-d^{2 t-n-1}+d^{2 t-n-2}-1 .
\end{aligned}
$$


Case 3b If $r \leq 2 t-n-3$, then, setting $q_{0}=\left\lfloor\frac{n+r+1}{2}\right\rfloor$, we obtain

$$
\begin{aligned}
\nu(G) & \leq\left|C_{q_{0}}\right| \leq d^{\left\lfloor\frac{n+r+1}{2}\right\rfloor}-1+k\left(d^{n-\left\lfloor\frac{n+r+1}{2}\right\rfloor}-1\right) \\
& \leq d^{\left\lfloor\frac{n+r+1}{2}\right\rfloor}-1+\left(d^{r+1}-1\right)\left(d^{n-\left\lfloor\frac{n+r+1}{2}\right\rfloor}-1\right) \\
& =d^{\left\lceil\frac{n+r+1}{2}\right\rceil}+d^{\left\lfloor\frac{n+r+1}{2}\right\rfloor}-d^{r+1}-d^{n-\left\lfloor\frac{n+r+1}{2}\right\rfloor .}
\end{aligned}
$$

The function

$$
g(r):=d^{\left\lceil\frac{n+r+1}{2}\right\rceil}+d^{\left\lfloor\frac{n+r+1}{2}\right\rfloor}-d^{r+1}-d^{n-\left\lfloor\frac{n+r+1}{2}\right\rfloor}
$$

is non-decreasing in $r$ when $0 \leq r \leq 2 t-n-3$, hence

$$
\nu(G) \leq g(2 t-n-3)=2 d^{t-1}-d^{2 t-n-2}-d^{n-t+1} .
$$

The upperbound of $\nu(G)$ in case $3 \mathrm{~b}$ is smaller than those in cases 2 and 3a (which are equal). Hence, when $2 t>n$

$$
\nu(G) \leq d^{t}-d^{2 t-n-1}+d^{2 t-n-2}-1 .
$$

Putting inequalities (3.1), (3.2), (3.3), and (3.4) together, we get

$$
|S| \leq \begin{cases}d^{n-2 t}+t d^{n-t}(d-1)-1 & \text { when } t<n / 2, \\ d^{n-t}[(d-1)(n-t)-1]+d^{t} & \text { when } t=n / 2, \\ d^{n-t}[(d-1)(n-t)-1]+d^{t}-d^{2 t-n-2}(d-1) & \text { when } t>n / 2\end{cases}
$$

The theorem follows because one more $\mathrm{BY}^{-1}(n)$ plane is needed to route the new request $(\mathbf{a}, B)$.

We next illustrate the strength of Theorem 3.1 by deriving as a consequence several known results.

In [26], necessary and sufficient conditions for $\log _{d}(N, 0, m)$ to be SNB under fanout constraints were proved. If the input stage (the demux stage) does not have fanout capability, then every multicast request must be routed entirely through the same Banyan plane. This corresponds to the window size $d^{n}$. It is crucial to notice that our analysis does not at all depend on how the requests were routed, as long as branches belonging to the same window are routed through the same plane. Hence, when the window size is $d^{n}$ we actually have a SNB condition under the constraint that the input stage does not have fanout capability. The following corollary matches the necessary and sufficient condition derived in [26]. We only need to "plug-in" the value $t=n$ in Theorem 3.1.

Corollary 3.2. If the input (demux) stage does not have fanout capability, then $\log _{d}(N, 0, m)$ is strictly nonblocking if $m \geq d^{n}-d^{n-1}+d^{n-2}$.

Next, when both stages have fanout capability, each unicast branch of a multicast request can be routed independently from each other. This corresponds exactly to the case when the window size is 1 , i.e. $t=0$. Similarly, our result gives a matching condition as the necessary and sufficient condition in [26]. 
Corollary 3.3. When both the demux stage and the Banyan stage have fanout capability, the $\log _{d}(N, 0, m)$ network is strictly non-blocking if $m \geq d^{n}$.

The previous two corollaries illustrate a counter-intuitive point: when the network has less capability, it might also cost less in the SNB sense. This is because being SNB requires the network to be able to find a new route for any existing network state, no matter how peculiar. The more capability the network has, the easier it is to construct a network state where more planes are blocking planes!

Last but not least, Theorem 3.1 allows for computing the best window size minimizing the number of switching elements in the network. A little calculus reveals that asymptotically picking $t \approx\left(n+\log _{d} n\right) / 2$ is best. In this case, the number of Banyan planes is $\Theta\left(n d^{n / 2}\right)=\Theta(\sqrt{N \log N})$. Each Banyan planes has depth $O(\log N)$ and crosspoint complexity $O(N \log N)$. In our optical switching sense, crosspoint complexity corresponds roughly to the number of directional couplers.

Corollary 3.4. Using multi-log networks, it is possible to construct $N \times N$ multicast WSNB crosstalk-free switching networks with depth $O(\log N)$ crosspoint complexity $\Theta\left((N \log N)^{3 / 2}\right)$.

This complexity is within a sub-linear factor of the optimal, known in the non-crosstalkfree case to be $\Theta(N \log N)$ ) but existing optimal constructions require larger depths [16].

\section{Concluding Remarks}

When there is no crosstalk-free constraint, only link-blocking takes effect. We have the following analogue of Proposition 2.1.

Proposition 4.1. Let $(\mathbf{a}, \mathbf{b})$ and $(\mathbf{x}, \mathbf{y})$ be two unicast requests. Then their corresponding routes $R(\mathbf{a}, \mathbf{b})$ and $R(\mathbf{x}, \mathbf{y})$ in (the same copy of) $B Y^{-1}(n)$ share at least a common link if and only if

$$
\left|\operatorname{SUF}\left(\mathbf{a}_{1 . . n-1}, \mathbf{x}_{1 . . n-1}\right)\right|+\left|\operatorname{PRE}\left(\mathbf{b}_{1 . . n-1}, \mathbf{y}_{1 . . n-1}\right)\right| \geq n .
$$

Following the proof of Theorem 3.1, define $S$ similarly and note that each $(\mathbf{x}, \mathbf{y}) \in S$ must belong to some $A_{i} \times B_{j}$ where $i+j \geq n$ (note the lowerbound of $n$ instead of $n-1$ as in Theorem 3.1). We partition $S$ into two parts $S_{1}$ and $S_{2}$ as before. The part $S_{1}$ is now bounded by

$$
\left|S_{1}\right| \leq \begin{cases}d^{n-1-2 t}-d^{t}+t d^{n-t-1}(d-1) & t<\frac{n-1}{2} \\ d^{n-t-1}(d-1)(n-t-1)-d^{n-t-1}+1 & t \geq \frac{n-1}{2}\end{cases}
$$

The $S_{2}$-part is bounded by

$$
\left|S_{2}\right| \leq \begin{cases}d^{t}-1 & t \leq n / 2 \\ d^{t}+d^{2 t-n-1}-d^{2 t-n}-1 & t>n / 2\end{cases}
$$


Noting $|S|=\left|S_{1}\right|+\left|S_{2}\right|$ and combining (4.2) and (4.3) we have given another proof of the following known result, which was derived recently in [2] with a much longer argument.

Theorem 4.2. The d-ary multi-log network $\log _{d}(N, 0, m)$ is widesense nonblocking under the window algorithm with window size $d^{t}$ if the number $m$ of Banyan planes is at least

$$
\left\{\begin{array}{l}
d^{n-1-2 t}+t d^{n-t-1}(d-1), \text { when } t<\frac{n-1}{2}, \\
d^{n-t-1}(d-1)(n-t-1)-d^{n-t-1}+d^{t}+1, \text { when } t=\frac{n}{2}, \\
d^{n-t-1}[(d-1)(n-t-1)-1]+d^{t}-d^{2 t-n-1}(d-1)+1, \\
\quad \text { when } t>n / 2
\end{array}\right.
$$

\section{References}

[1] Chinni, V., Huang, T., Wai, P.-K., Menyuk, C., And Simonis, G. Crosstalk in a lossy directional coupler switch. J. Lightwave Technol. 13, 7 (1995), 1530-1535.

[2] DANILEWICZ, G. Wide-sense nonblocking $\log _{d}(n, 0, p)$ multicast switching networks. IEEE Transactions on Communications 55, 11 (2007), 2193-2200.

[3] D.LI. Elimination of crosstalk in directional coupler switches. Optical Quantum Electron., 25, 4 (1993), 255-260.

[4] F.K.HWANG, AND Lin, B.-C. Wide-sense nonblocking multicast $\log _{2}(n, m, p)$ networks. IEEE Transactions on Communications 51, 10 (Oct 2003), 1730-1735.

[5] HWANG, F. Choosing the best $\log _{2}(n, m, p)$ strictly nonblocking networks. IEEE Transactions on Communications 46, 12 (Dec 1998), 454-455.

[6] HwANG, F. K. The mathematical theory of nonblocking switching networks. World Scientific Publishing Co. Inc., River Edge, NJ, 1998.

[7] Jiang, X., Pattavina, A., And S.Horiguchi. Rearrangeable $f$-cast multi- $\log 2 n$ networks. IEEE Transactions on Communications (2007). to appear.

[8] Jiang, X., Shen, H., UR RAShid KhandKer, M. M., AND Horiguchi, S. Blocking behaviors of crosstalk-free optical banyan networks on vertical stacking. IEEE/ACM Transactions on Networking 11, 6 (2003), 982-993.

[9] KabaCinSKI, W., AND DANILEWICZ, G. Wide-sense and strict-sense nonblocking operation of multicast multi- $\log _{2} n$ switching networks. IEEE Transactions on Communications 50, 6 (Jun 2002), 1025-1036.

[10] LEA, C.-T. Muti- $\log _{2} n$ networks and their applications in high speed electronic and photonic switching systems. IEEE Transactions on Communications 38, 10 (1990), 1740-1749.

[11] LEA, C.-T., AND SHYY, D.-J. Tradeoff of horizontal decomposition versus vertical stacking in rearrangeable nonblocking networks. IEEE Transactions on Communications 39 (1991), 899904.

[12] Lovász, L., AND Plummer, M. D. Matching theory. North-Holland Publishing Co., Amsterdam, 1986. Annals of Discrete Mathematics, 29.

[13] Maier, G., And Pattavina, A. Design of photonic rearrangeable networks with zero firstorder switching-element-crosstalk. IEEE Trans. Comm. 49, 7 (Jul 2001), 1248-1279.

[14] Mukherjee, B. Optical Communication Networks. McGraw-Hill, New York, NY, 1997.

[15] NGO, H. Q. WDM switching networks, rearrangeable and nonblocking $[w, f]$-connectors. SIAM Journal on Computing 35, 3 (2005-2006), 766-785.

[16] NGO, H. Q., AND Du, D.-Z. Notes on the complexity of switching networks. In Advances in Switching Networks, D.-Z. Du and H. Q. Ngo, Eds., vol. 5 of Network Theory and Applications. Kluwer Academic Publishers, 2001, pp. 307-367. 
[17] NGO, H. Q., PAN, D., AND QIAO, C. Nonblocking WDM switches based on arrayed waveguide grating and limited wavelength conversion. In Proceedings of the 23rd Conference of the IEEE Communications Society (INFOCOM) (Hong Kong, China, 2004), IEEE.

[18] NGO, H. Q., PAN, D., AND YANG, Y. Optical switching networks with minimum number of limited range wavelength converters. In Proceedings of the 24rd Annual Joint Conference of the IEEE Computer and Communications Societies (INFOCOM) (Miami, Florida, U.S.A., March 2005), vol. 2, IEEE, pp. 1128-1138.

[19] NGO, H. Q., WAng, Y., LE, A., AND JiAnG, X. Better necessary conditions for rearrangeably nonblocking f-cast d-ary multi-log networks under fanout and crosstalk constraints. In Proceedings of the 2008 International Workshop on High Performance and Highly Survivable Routers and Networks (HPSRN) (Sendai, Japan, Mar 2008), IEEE.

[20] QIaO, C., AND Yoo, M. Optical burst switching (OBS) - a new paradigm for an optical internet. Journal of High Speed Networks 8, 1 (1999), 69-84.

[21] Shyy, D.-J., AND LEA, C.-T. $\log _{2}(n, m, p)$ strictly nonblocking networks. IEEE Transactions on Communications 39, 10 (1991), 1502-1510.

[22] Stern, T. E., AND BALA, K. Multiwavelength Optical Networks: A Layered Approach. Prentice Hall PTR, Upper Saddle River, NJ, 1999.

[23] TsChA, Y., AND LEE, K.-H. Yet another result on multi- $\log _{2} n$ networks. IEEE Transactions on Communications 47, 9 (Sep 1999), 1425-1431.

[24] VAEZ, M., AND LEA, C.-T. Wide-sense nonblocking Banyan-type switching systems based on directional couplers. IEEE J. Select. Areas Commun. 16, 7 (Sep 1998), 1327-1332.

[25] VAEZ, M. M., AND LEA, C.-T. Strictly nonblocking directional-coupler-based switching networks under crosstalk constraint. IEEE Trans. Comm. 48, 2 (Feb 2000), 316-323.

[26] WANG, Y., NGO, H. Q., AND JIANG, X. Strictly nonblocking $f$-cast $d$-ary multilog networks under fanout and crosstalk constraints. In Proceedings of the 2008 International Conference on Communications (ICC) (Bejing, China, 2008), IEEE.

[27] WU, J.-C., AND TSAI, T.-L. Low complexity design of a wavelength-selective switch using raman amplifiers and directional couplers. In GLOBECOM (2006).

[28] Yang, Y., AND WANG, J. Designing WDM optical interconnects with full connectivity by using limited wavelength conversion. IEEE Trans. Comput. 53, 12 (2004), 1547-1556.

[29] YANG, Y., AND WANG, J. WDM optical switching networks using sparse crossbars. In Proceedings of the 23th Annual Joint Conference of the IEEE Computer and Communications Societies (INFOCOM'2004, Hong Kong) (2004), IEEE. 